\title{
Large Fermi surface expansion through anisotropic mixing of conduction and $f$ electrons in the semimetallic Kondo lattice CeBi
}

\author{
Peng Li, ${ }^{1}$ Zhongzheng Wu, ${ }^{1}$ Fan Wu, ${ }^{1}$ Chunyu Guo, ${ }^{1}$ Yi Liu, ${ }^{2}$ Haijiang Liu,${ }^{3}$ Zhe Sun, ${ }^{2}$ Ming Shi, ${ }^{3}$ Fanny Rodolakis,${ }^{4}$ \\ Jessica L. McChesney, ${ }^{4}$ Chao Cao ${ }^{5}$ Huiqiu Yuan, ${ }^{1,6,7,{ }^{*}}$ Frank Steglich, ${ }^{1, \dagger}$ and Yang Liu $\oplus^{1,6,7,+}$ \\ ${ }^{1}$ Center for Correlated Matter and Department of Physics, Zhejiang University, Hangzhou 310058, People's Republic of China \\ ${ }^{2}$ National Synchrotron Radiation Laboratory, University of Science and Technology of China, Hefei 230026, People's Republic of China \\ ${ }^{3}$ Paul Scherrer Institute, Swiss Light Source, CH-5232 Villigen PSI, Switzerland \\ ${ }^{4}$ Advanced Photon Source, Argonne National Laboratory, 9700 South Cass Avenue, Argonne, Illinois 60439, USA \\ ${ }^{5}$ Department of Physics, Hangzhou Normal University, Hangzhou 311121, People's Republic of China \\ ${ }^{6}$ Zhejiang Province Key Laboratory of Quantum Technology and Device, Zhejiang University, Hangzhou 310058, People's Republic of China \\ ${ }^{7}$ Collaborative Innovation Center of Advanced Microstructures, Nanjing University, Nanjing 210093, People's Republic of China
}

(Received 14 June 2019; revised manuscript received 16 September 2019; published 7 October 2019)

\begin{abstract}
Using angle-resolved photoemission spectroscopy (ARPES) and resonant ARPES, we report evidence of strong anisotropic conduction- $f$ electron mixing ( $c-f$ mixing) in $\mathrm{CeBi}$ by observing a largely expanded Ce $5 d$ pocket at low temperature, with no change in the $\mathrm{Bi} 6 p$ bands. The anisotropic Fermi surface (FS) expansion is accompanied by a pronounced spectral weight transfer from the local $4 f^{0}$ peak of $\mathrm{Ce}$ (corresponding to $\mathrm{Ce}^{3+}$ ) to the itinerant conduction bands near the Fermi level. Careful analysis suggests that the observed large FS change (with a volume expansion of the electron pocket up to $40 \%$ ) can most naturally be explained by a small valence change $(\sim 1 \%)$ of $\mathrm{Ce}$, which coexists with a very weak Kondo screening. Our work therefore provides evidence for a FS change driven by real charge fluctuations deep in the Kondo limit, which is highly dependent on the orbital character and momentum and is made possible by the low carrier density.
\end{abstract}

DOI: $10.1103 /$ PhysRevB.100.155110

\section{INTRODUCTION}

Intermetallic compounds of some rare earths (REs, notably $\mathrm{Ce}$ and $\mathrm{Yb}$ ) frequently behave as Kondo-lattice (KL) systems. Well below the Kondo temperature $T_{K}$, they can develop different types of interesting ground-state properties, including heavy Fermi-liquid, unconventional superconducting, magnetically ordered, or Kondo insulating/semimetallic phases [1-5]. For these materials, $T_{K}$ may vary between a few $\mathrm{K}$ ("Kondo limit") and a few hundred K ["intermediate valence (IV) limit"]. In the Kondo limit, Kondo screening of the local $4 f$ moments plays the key role over a wide temperature range up to above $T_{K}$. This is a many-body process involving the local $4 f$ electron states and electron-hole pairs at the Fermi level $\left(E_{F}\right)$, leading to spin flips without changing the $4 f$ occupation ("virtual charge fluctuations"). Meanwhile, there exists a minority of "real charge (valence) fluctuations" due to the weak (covalent) mixing or hybridization, which is necessary for the Kondo screening to operate, as described by the periodic Anderson model [6]. Therefore, for KL systems, the Ce valence is not exactly integer (typically $3<v<3.1$ ) and increases slightly upon cooling. In the IV limit, where $v$ is typically between 3.2 and 3.5 , the valence fluctuations predominate, but the Kondo spin flips are still operating. This is illustrated by the negative temperature coefficient (NTC)

\footnotetext{
*hqyuan@zju.edu.cn

${ }^{\dagger}$ Frank.Steglich@cpfs.mpg.de

†yangliuphys@zju.edu.cn
}

of the resistivity, $\rho(T) \sim-\log T / T_{K}$, in the canonical IV compound $\mathrm{CePd}_{3}$ [7]. On the other hand, it was found for dilute IV Eu ions in $\mathrm{ScAl}_{2}$ in the absence of Kondo spin flips that the incremental Eu-derived resistivity exhibits a positive $T$ coefficient [8], while a Kondo-type NTC is observed for a few Eu-based compounds [9]. It is therefore a big challenge to resolve valence fluctuations from Kondo spin flips, particularly for KL systems with low $T_{K}$, where the Kondo screening is predominant, and the effect of tiny valence fluctuations is almost impossible to identify unambiguously in transport and thermodynamic measurements.

As will be shown below, low carrier density KL systems provide an interesting case to resolve valence-fluctuationinduced changes in the Fermi surface (FS), deep in the Kondo limit. The low carrier density significantly reduces the Kondo screening, while the small FS allows for easy detection of any change in the Fermi wave vector $k_{F}$ due to valence fluctuations. Low carrier density Kondo systems have attracted considerable interest recently, in view of novel phenomena such as unconventional quantum criticality and correlated topological states [10-14]. This has motivated us to study $\mathrm{CeBi}$, a well-known low carrier density $\mathrm{KL}$ system $\left(\sim 0.03 e^{-} / \mathrm{Ce}[15]\right) . \mathrm{CeBi}$ was extensively studied in the past because of its anomalous electromagnetic properties [16-21]. Its resistivity shows NTC suggesting Kondo screening, with a very low $T_{K}$ due to the few carriers available. As a consequence, the $4 f$ moments are only very weakly screened and undergo antiferromagnetic (AFM) order at $T_{\mathrm{N} 1} \simeq 25 \mathrm{~K}$ and $T_{\mathrm{N} 2} \simeq 12$, respectively [16,22]. According to Kasuya and collaborators $[17,23]$, the weak Kondo interaction becomes 
substantially strengthened by the strong mixing between the Bi $6 p$ and Ce $4 f$ states ( $p$ - $f$ mixing). An alternative proposal, however, has argued for non-Kondo exchange to explain the electromagnetic properties [18]. The band structure of $\mathrm{CeBi}$ was studied previously by angle-resolved photoemission spectroscopy (ARPES) [14,19], revealing the coexistence of hole and electron bands near $E_{F}$, but the low-temperature properties, particularly the role of $4 f$ electrons, were not addressed.

\section{EXPERIMENTAL AND COMPUTATIONAL DETAILS}

ARPES measurements of single-crystal CeBi (with a typical size of $\sim 1 \times 1 \mathrm{~mm}$ ) were performed at low temperatures (from 10 to $\sim 70 \mathrm{~K}$ ), after being cleaved in situ at low temperatures with a base pressure $<1 \times 10^{-10}$ Torr. Off-resonant ultraviolet ARPES measurements $(h v<40 \mathrm{eV})$ were carried out at the BL13U beamline in National Synchrotron Radiation Lab (NSRL, Hefei). The Fermi surface (FS) maps are obtained using a DA30 electron analyzer with deflector scanning mode. On-resonant ARPES measurements near the Ce $N(\sim 122 \mathrm{eV})$ edge were performed at the SIS beamline at the Swiss Light Source, Villigen. In order to probe the intrinsic bulk electronic structure, soft x-ray resonant ARPES measurements were also performed near the Ce $M$ edge $(\sim 880 \mathrm{eV})$ at the 29 -ID IEX beamline at the Advanced Photon Source, Argonne. The typical energy (momentum) resolution is $\sim 15 \mathrm{meV}\left(\sim 0.01 \AA^{-1}\right)$ for both the ultraviolet off-resonant and the $N$ edge resonant ARPES, while the energy (momentum) resolution is $\sim 100 \mathrm{meV}\left(\sim 0.03 \AA^{-1}\right)$ for the $M$ edge resonant ARPES. The much reduced energy/momentum resolution in the $M$ edge resonant ARPES is due to the large photon (and hence photoelectron) energy, which imposes technical challenges in these measurements. Nevertheless, the $M$ edge resonant ARPES measurement is much more bulk sensitive and predominantly probes the bulk Ce $4 f$ electrons, which provides important orbital-specific information. All data presented in this paper were taken only within a few hours after the low-temperature cleave (typically $<5 \mathrm{~h}$ ), to ensure that our results are not affected by sample aging.

Electronic structure calculations in this paper were performed using density functional theory (DFT) and a planewave basis projected augmented wave method, as implemented in the Vienna $A b$ Initio Simulation Package (VASP) [24]. $f$ electrons have been treated as core electrons and spin-orbit coupling is taken into account in the calculations [25]. We have employed the modified Becke-Johnson (MBJ) potential to obtain good agreement with experiments [26,27]. The spectral calculations in Figs. 1(b) and 1(c) are obtained from the surface Green's function (GF) method using a Hamiltonian from DFT calculations [25]. This method integrates the states from semi-infinite layers and the surface. Numerically, the infinitesimal imaginary number was chosen to be $\eta=$ $0.001 \mathrm{eV}$ to ensure causality in the retarded GF.

\section{RESULTS AND DISCUSSION}

\section{A. Band structure and Fermi surface expansion}

Bulk CeBi crystallizes in the simple rocksalt structure; its three-dimensional (3D) Brillouin zone (BZ) and associated two-dimensional (2D) BZ are shown in Fig. 1(a). At high temperature, the $\mathrm{Ce}$ has a valence close to +3 (with one $4 f$ electron), and the FS consists of two small hole pockets at the $\Gamma$ point (from Bi $6 p$ orbitals) and one small electron pocket at each symmetry-equivalent $X$ point (from Ce $5 d$ orbitals) [14,27]. All states contributing to the FS are bulk states. The bottom panel in Fig. 1(a) displays the 3D bulk FS from density-functional theory (DFT) calculations, assuming the $4 f$ electrons to be completely localized [25,27]. The experimental FSs near the $2 \mathrm{D} \bar{\Gamma}$ and $\bar{M}$ points (the $X$ point in 3D bulk BZ projects onto the $\bar{M}$ point in 2D BZ), obtained by ARPES measurements at both $10 \mathrm{~K}$ and $\sim 37 \mathrm{~K}$, are displayed in Figs. 1(b) and 1(c), in comparison with the $k_{z}$ projected DFT calculations. Good overall agreements between the experiments and calculations can be found, implying that the $4 f$ electrons remain almost fully localized (hence excluded from the FS) even down to $\sim 10 \mathrm{~K}$.

Assuming completely localized $4 f$ electrons, the Luttinger volume of the hole pockets ( $\mathrm{Bi} 6 p$ ) should be the same as that of the electron pockets ( $\mathrm{Ce} 5 d$ ), as required by the charge neutralization. However, we observe experimentally that the electron pocket near the $\bar{M}$ point expands considerably at lower temperatures, while the hole pockets near the $\bar{\Gamma}$ point remain unchanged [see momentum-distribution curves (MDCs) in Figs. 1(d) and 1(e) and energy-momentum cuts in Figs. 2(a) and 2(b)]. Our detailed analysis indicates that the electron pocket expands mainly along the short axis [Fig. 1(e)], which results in a volume increase of $\sim 40 \%$ at $\sim 10 \mathrm{~K}$ compared to that at $\sim 37 \mathrm{~K}$, while no change can be detected for the hole pocket. This temperature dependence is confirmed by measurements from different samples $(>10)$. The imbalanced change of hole and electron pockets implies that additional electrons (other than $\mathrm{Bi} 6 p$ and $\mathrm{Ce} 5 d$ ) become involved in the FS at low temperature.

\section{B. Temperature evolution of the electron pocket}

The temperature evolution of $k_{\mathrm{F}}$ for the electron pocket is summarized in Fig. 2(c), where the top panel shows the raw data from one representative sample, demonstrating the onset temperature of $\sim 35 \mathrm{~K}$ for the pocket expansion. To account for slight variations in $k_{\mathrm{F}}$ for different samples and highlight the temperature evolution, we normalize the $k_{\mathrm{F}}$ with respect to its high temperature value, i.e., $k_{\mathrm{F}}(T) / k_{\mathrm{F}}(T>35 \mathrm{~K})$, and plot it at the bottom panel for four different samples. The result clearly shows that the pocket gradually expands with decreasing temperature and eventually reaches a plateau near $10 \mathrm{~K}$. Since CeBi develops AFM order at $\sim 25 \mathrm{~K}$, the observed FS expansion might be related to the AFM transition. However, neither the FS maps in Fig. 1 nor the energy-momentum cuts in Figs. 2(a) and 2(b) show any sign of band folding at low temperature (more data can be found in the Supplemental Material [28]), indicating that the band folding effect from AFM ordering is very weak and does not play a significant role here. We also performed similar ARPES measurements for GdBi [Fig. 2(d)], an isostructural compound with AFM ordering at $25 \mathrm{~K}$; the results show no temperature dependence for both the hole and electron pockets, in sharp contrast to CeBi (see the Supplemental Material [28] for additional data 
(a)
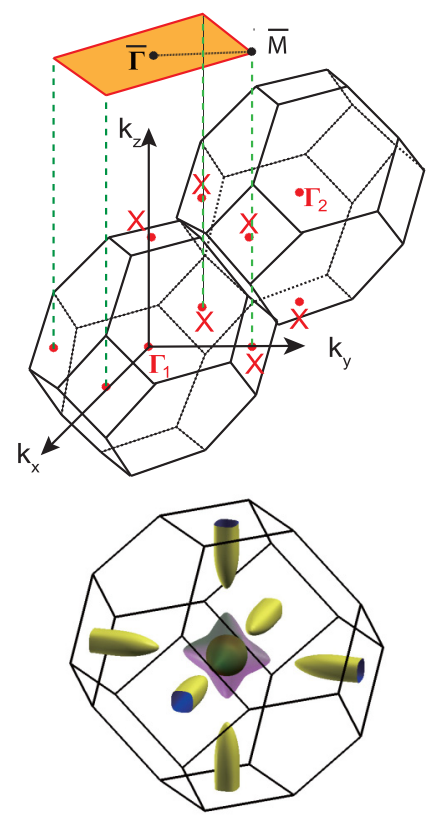

(b)
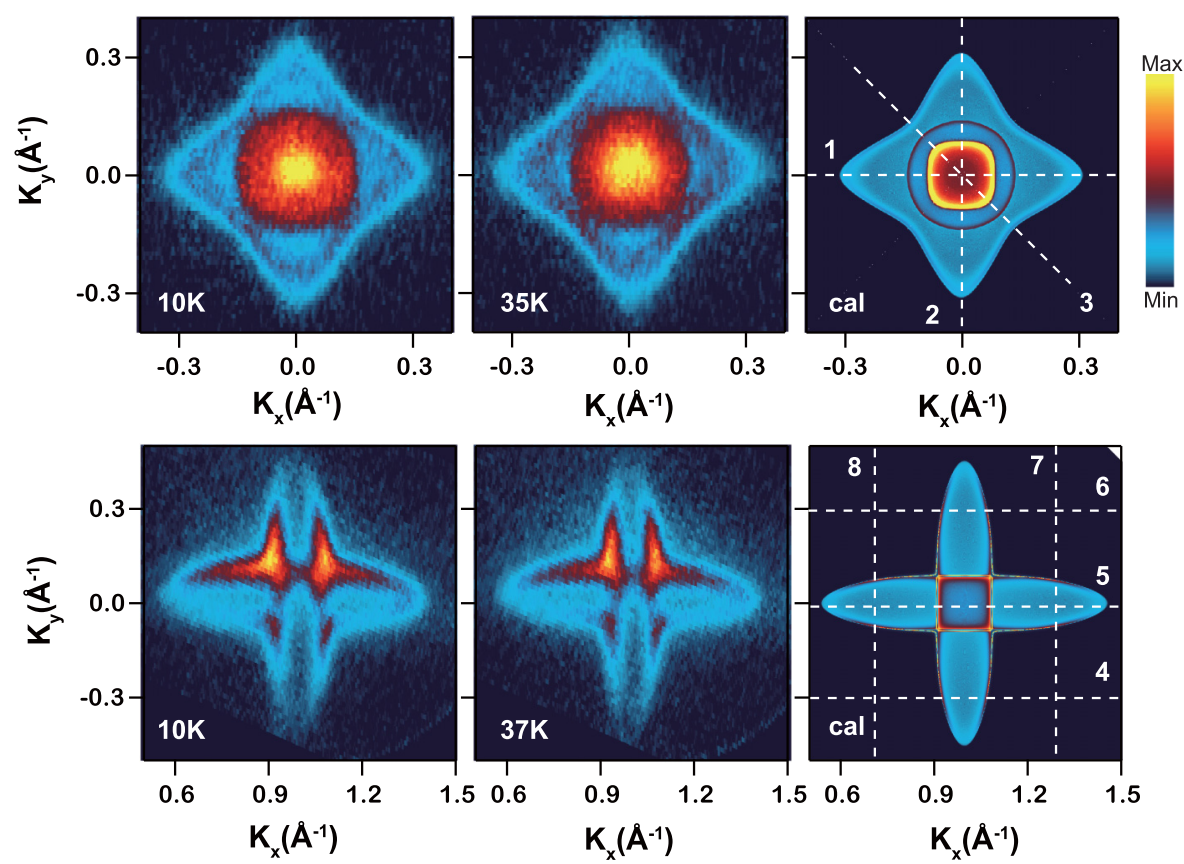

(d)
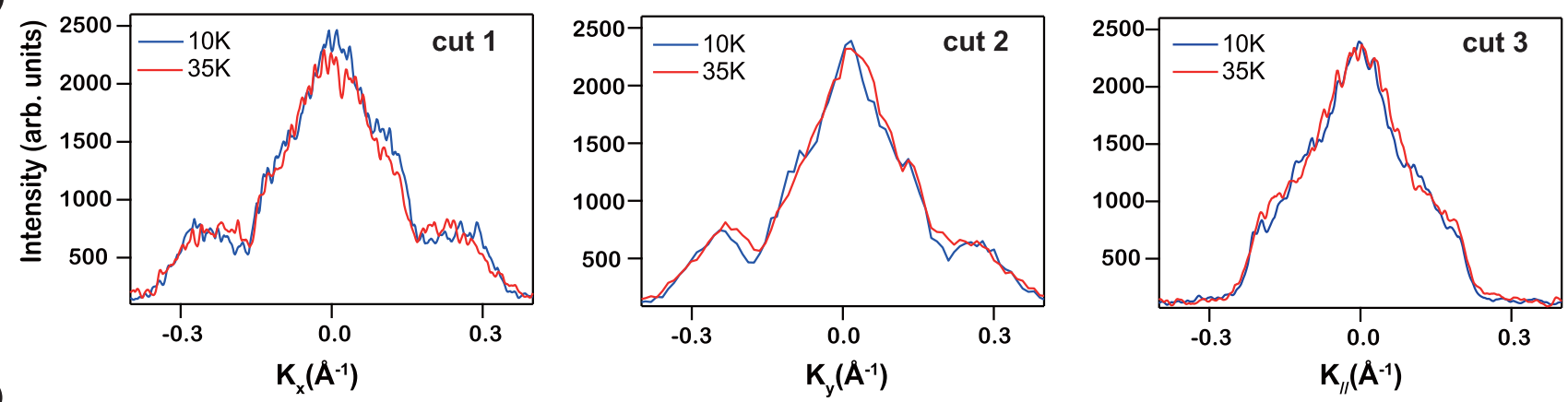

(e)
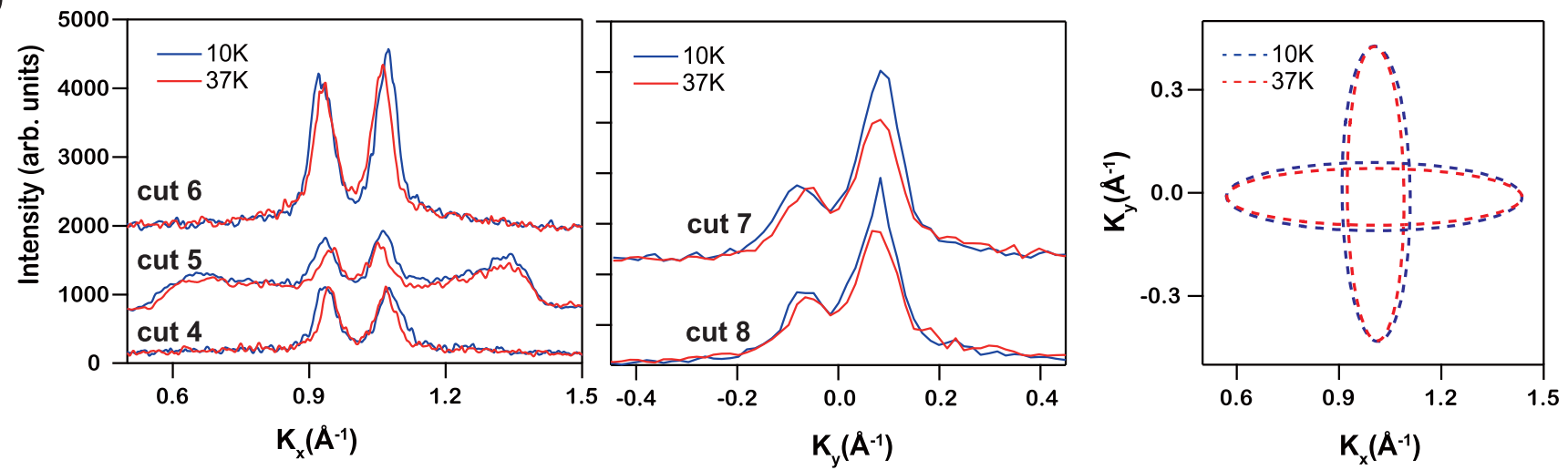

FIG. 1. Temperature-dependent FS of CeBi. (a) 3D and projected 2D BZs of CeBi (top), and the calculated 3D FS excluding Ce $4 f$ electrons (bottom). (b,c) Experimental FS at 10 and $\sim 37 \mathrm{~K}$ (left and middle panels), in comparison with the DFT calculations (right panels, denoted by calc), for both the hole pocket near the $\bar{\Gamma}$ point (b) and the electron pocket near the $\bar{M}$ point (c). The DFT calculations contain spectral contributions from all $k_{z}$ 's in the 3D BZ, as well as a small energy broadening $(0.001 \mathrm{eV})$. (d,e) MDC cuts for the hole (d) and electron (e) pockets. The cut directions are indicated in $(\mathrm{b}, \mathrm{c})$. The rightmost panel in (e) shows the extracted contour of the electron pocket at low and high temperatures.

and analysis). In contrast to the "unstable" $4 f$ shell of CeBi with $4 f$ occupancy $n_{f} \leqslant 1$, the half-filled one $\left(n_{f}=7\right)$ of GdBi is very "stable." Therefore, the observed expansion of the electron pocket in $\mathrm{CeBi}$ should most naturally be associated with the Ce-derived $4 f$ electrons. This is also supported by the comparison of $\rho(T)$ in Fig. 2(e): While the resistivity of $\mathrm{CeBi}$ exhibits a clear Kondo-type upturn from high temperature to $\sim 25 \mathrm{~K}$ and a sharp decrease below $25 \mathrm{~K}$, the resistivity of GdBi shows only a tiny kink at the AFM transition, without any sign of Kondo-like behavior. For CeBi, the incremental resistivity, obtained after subtracting the result for $\mathrm{LaBi}\left(\rho_{\mathrm{i}}=\rho_{\mathrm{CeBi}}-\rho_{\mathrm{LaBi}}\right)$, indeed follows the logarithmic 
(a)

(b)
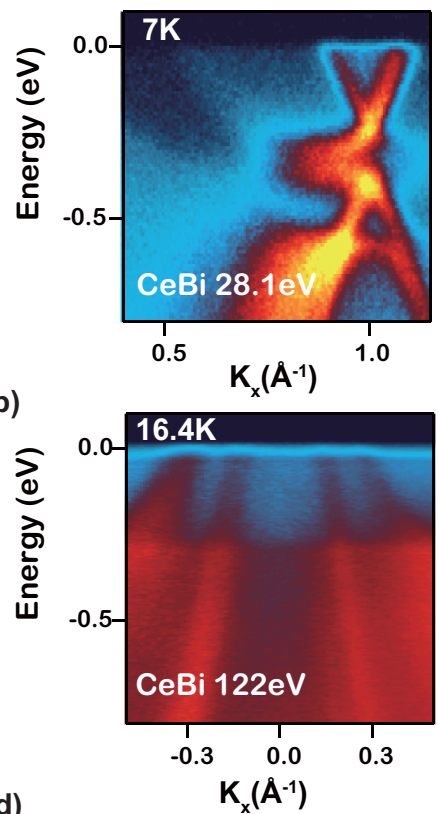

(d)

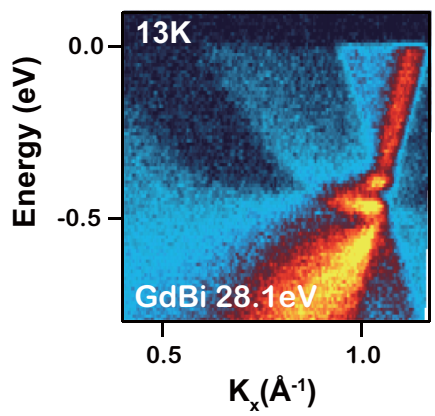

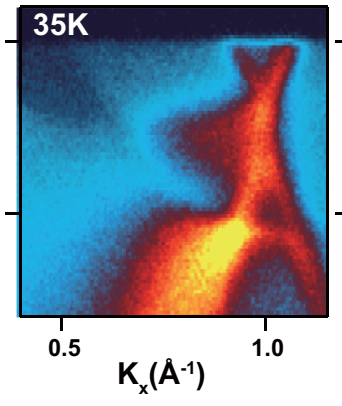
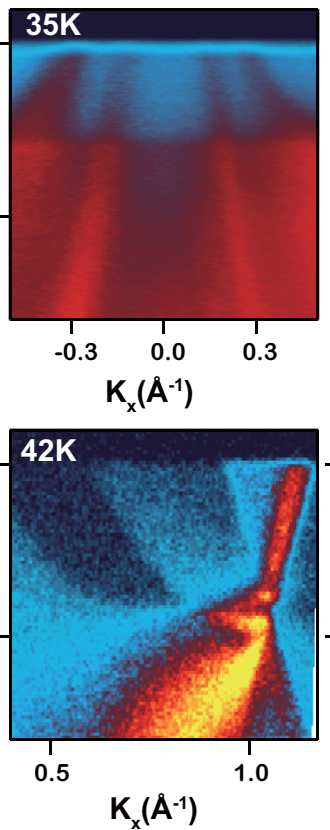
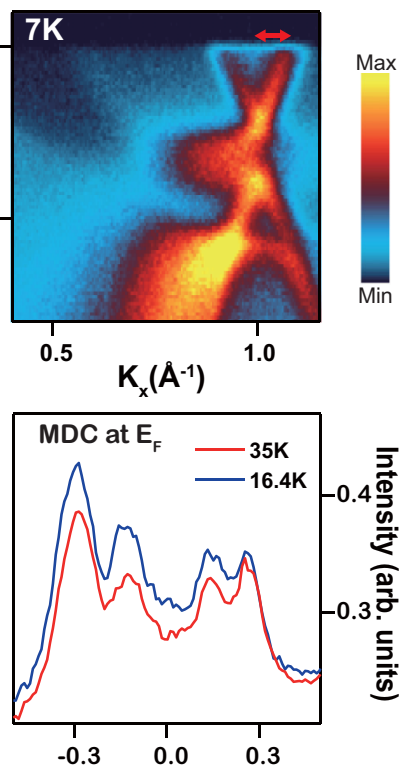

$K_{x}\left(\AA^{-1}\right)$

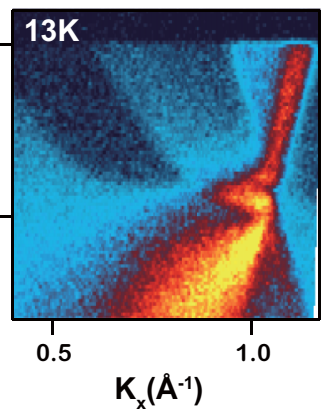

(c)
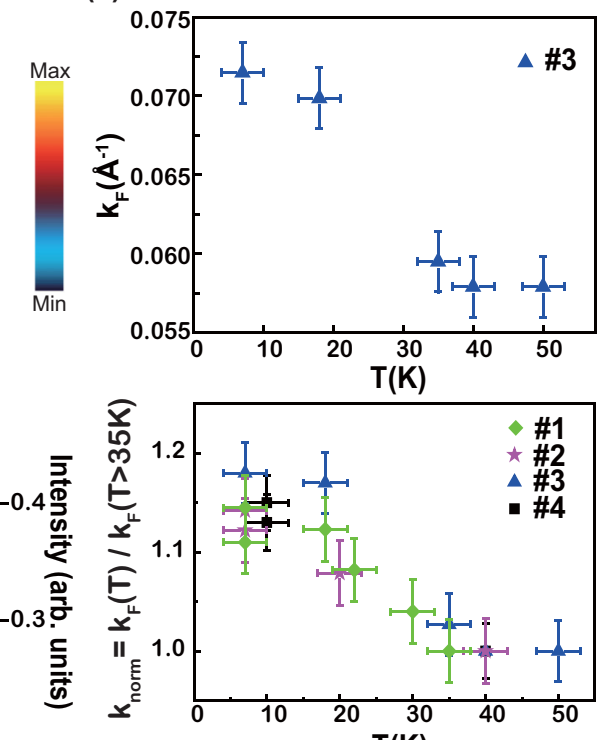

(e)

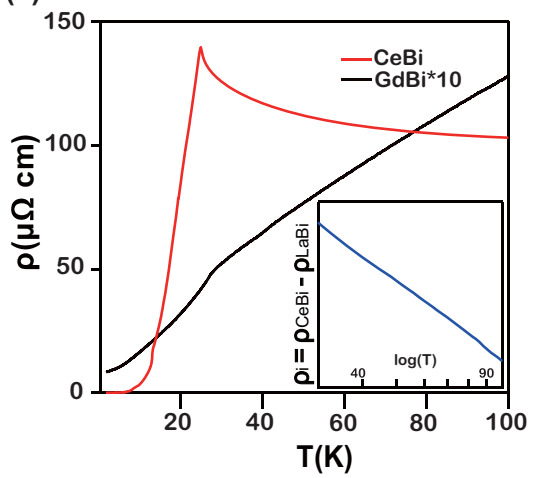

FIG. 2. Temperature evolution of the hole and electron pocket for $\mathrm{CeBi}$, in comparison with GdBi. (a,b) Temperature dependence of the energy-momentum cut for the electron (a) and hole (b) pocket. The data in (a) show the evolution during a warming and cooling cycle from the same sample. The rightmost panel in (b) shows the MDC cuts at $E_{\mathrm{F}}$; no change in $k_{\mathrm{F}}$ can be observed. (c) Temperature evolution of $k_{\mathrm{F}}$ for the electron pocket [indicated by a red arrow in (a)]. The top panel displays the raw data from a representative sample (\#3), showing the onset of pocket expansion at $\sim 35 \mathrm{~K}$. The bottom panel summarizes the normalized Fermi vector $k_{\text {norm }}=k_{\mathrm{F}}(T) / k_{\mathrm{F}}(T>35 \mathrm{~K})$, for different samples. (d) Temperature evolution of the electron pocket for $\mathrm{GdBi}$, showing no discernible change. (e) Resistivity vs temperature for CeBi and GdBi. The inset is the incremental part of the resistivity of $\mathrm{CeBi}, \rho_{\mathrm{i}}=\rho_{\mathrm{CeBi}}-\rho_{\mathrm{LaBi}}$, plotted as a function of $\log T$.

temperature dependence characteristic for Kondo scattering [inset of Fig. 2(e)].

\section{Temperature-dependent spectral weight transfer in resonant ARPES}

To better understand the Kondo effect and $c$ - $f$ mixing, we performed resonant ARPES measurements at Ce $N$ edge $(4 d \rightarrow 4 f$ ) to enhance the spectral contribution from $4 f$ electrons [Fig. 3(a)]. We can clearly observe a sharp dispersionless $4 f^{0}$ peak at $\sim-2.8 \mathrm{eV}$ and the $4 f^{1}$ peak at $-0.3 \mathrm{eV}$, respectively. Here the labels $4 f^{0}$ and $4 f^{1}$ refer to the electronic configuration after photoexcitation - therefore the $4 f^{0}$ and $4 f^{1}$ peaks correspond to the localized $4 f$ electron $\left(\mathrm{Ce}^{3+}\right)$ and the many-body Kondo resonance (KR), respectively. The $4 f^{1}$ peak at $-0.3 \mathrm{eV}$ is actually the spin-orbit split satellite (angular momentum $J=7 / 2$ ) of the KR, while the $J=5 / 2$ $\mathrm{KR}$ peak near $E_{F}$ is quite weak and almost absent [see
Figs. 2(b) and 3(a)], similar to CeSb [29]. The presence of the $J=7 / 2$ peak implies that the Kondo process is indeed active, and the rather weak $J=5 / 2$ peak indicates that its $T_{K}$ must be very low [30,31]. The small $T_{K}$ is well expected for a low carrier density KL system, as the strength of the Kondo screening is strongly dependent on the density of conduction electrons [1].

Figure 3(a) also shows the comparison of energydistribution curves (EDCs) near the hole and electron pockets at 16 and $35 \mathrm{~K}$, respectively. A very small decrease of the $4 f^{0}$ peak intensity and a slight increase of the conduction bands near the $J=7 / 24 f^{1}$ peak are seen at lower temperature. This trend becomes much clearer for soft x-ray resonant ARPES measurements at the Ce $M$ edge [Figs. 3(b) and 3(c)], which is more bulk sensitive due to higher energies. Below resonance $(875 \mathrm{eV})$, the APRES spectra are dominated by the non- $f$ conduction bands, whose EDCs exhibit no temperature dependence [Fig. 3(c)]. Upon cooling at resonance, e.g., at 
(a)

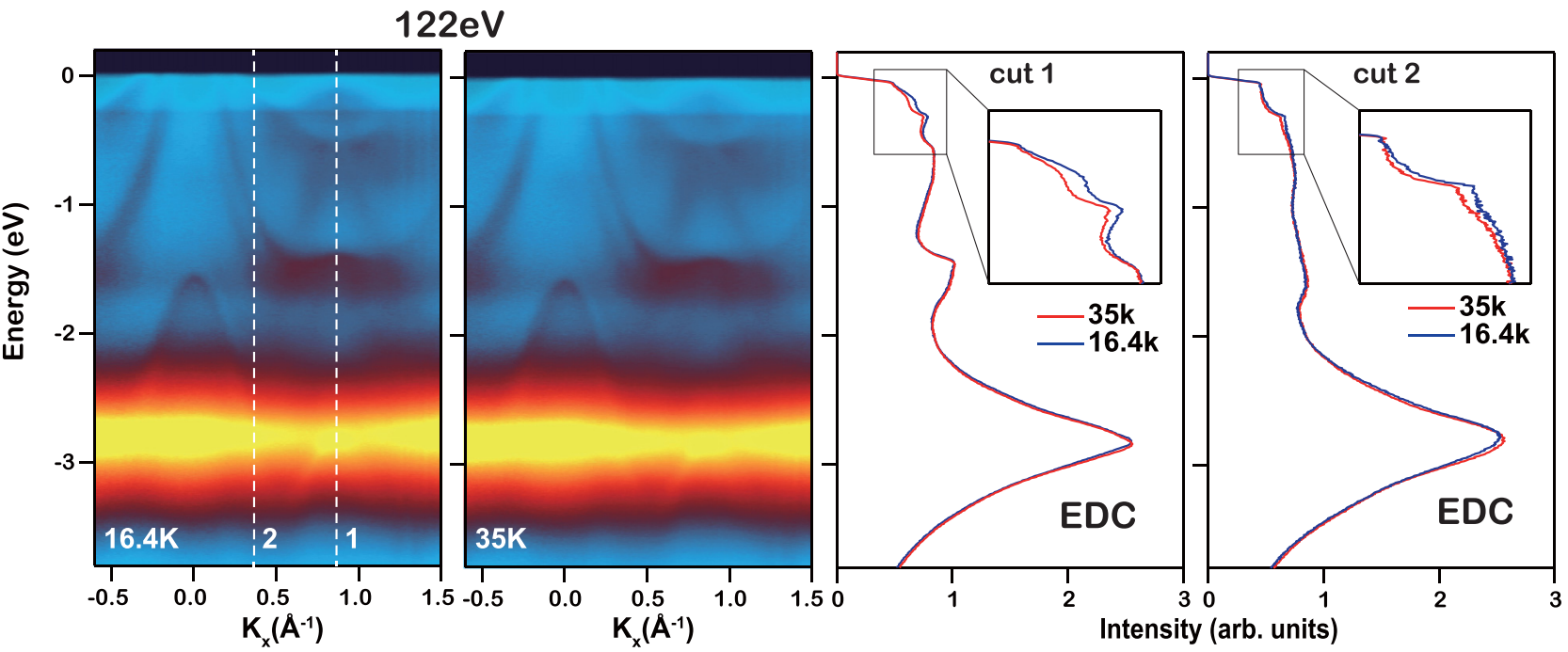

(b)
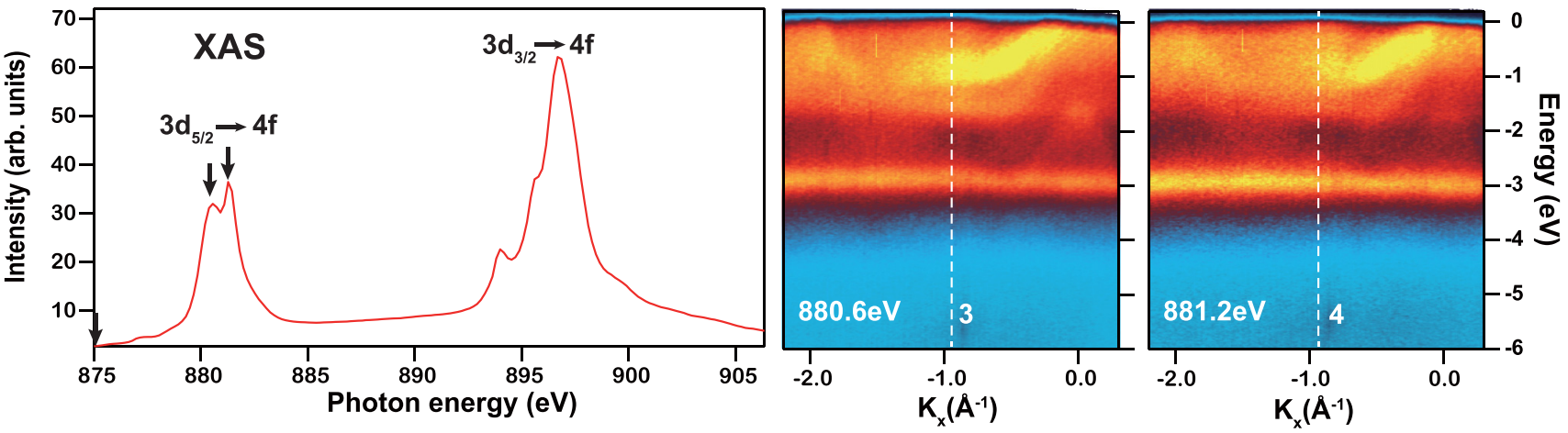

(c)

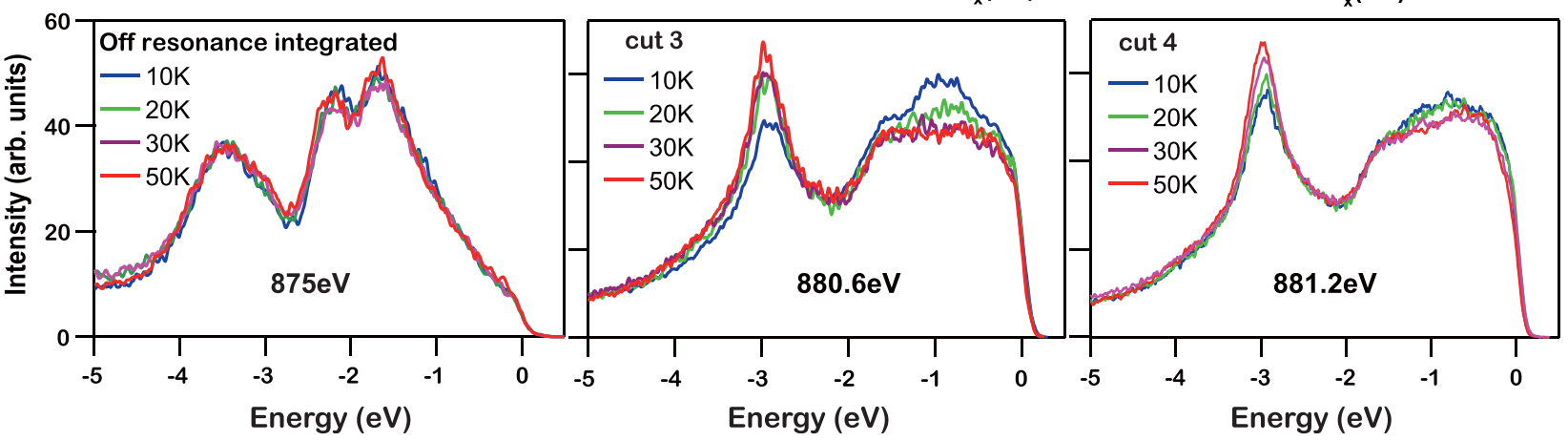

FIG. 3. Resonant ARPES results and temperature evolution. (a) Resonant ARPES results at Ce $N$ edge (122 eV) at two representative temperatures. The EDC cuts at the hole and electron pockets are shown on the right panels, respectively. (b) Left: X-ray absorption spectroscopy (XAS) data near Ce $M$ edge at $10 \mathrm{~K}$. Right: Soft x-ray ARPES spectra taken at two representative photon energies (880.6 and $899.4 \mathrm{eV}$ ) at $10 \mathrm{~K}$, indicated by black arrows in the XAS data. (c) Temperature dependence of EDCs at three representative photon energies (and momenta), indicated as white vertical lines in (b).

880.6 and $881.2 \mathrm{eV}$, one can clearly observe a pronounced decrease of the $4 f^{0}$ peak and a simultaneous intensity increase of the conduction bands from $E_{\mathrm{F}}$ to $\sim-1.5 \mathrm{eV}$. Additional data from another sample with a cooling and warming cycle are shown in the Supplemental Material [28]. Here the conduction bands are not resolved as clearly as those in Figs. 1 and 2, likely due to the limited resolution and low counts in soft x-ray ARPES. The pronounced temperature-dependent intensity transfer in Fig. 3(c) represents direct evidence for the strong $c-f$ mixing at low temperature, which couples the wave functions of conduction and $f$ electrons, resulting in higher $f$ weight near $E_{\mathrm{F}}$ (itinerant) and lower $f$ weight at $4 f^{0}$ (localized) with decreasing temperature.

\section{Valence-induced Fermi surface change}

Our observation that both the spectral weight transfer in Fig. 3(c) and the FS expansion in Fig. 2(c) onset at $\simeq 35 \mathrm{~K}$ indicate that these two phenomena are intimately connected. According to Luttinger's theorem, the transfer of (a small amount of localized) $4 f$ electrons into the conduction bands can lead to an expanded FS. Note that the FS change seen here is different from the small-to-large FS change observed in heavy fermion (HF) metals upon cooling [32-36], during which a strong and coherent KR develops near $E_{\mathrm{F}}$ and hybridizes with the conduction bands, resulting in an increased $k_{\mathrm{F}}$. Figure $4(\mathrm{a})$ shows the zoomed-in view of the ARPES 
(a)
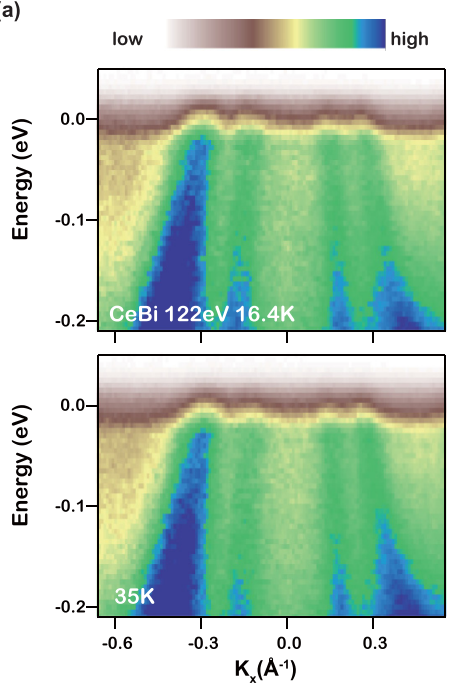

(b)

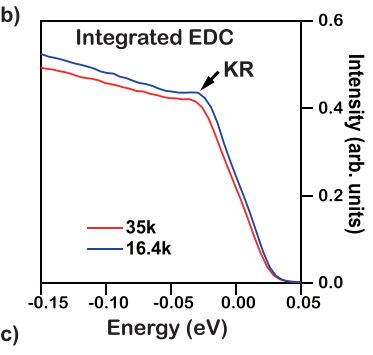

(c)

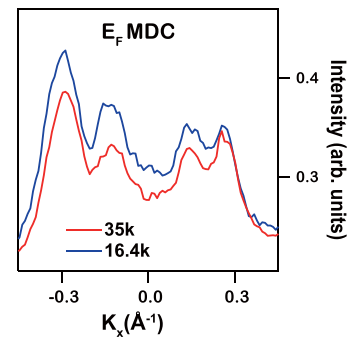

FIG. 4. (a) Zoomed-in view of the ARPES spectra at the $\bar{\Gamma}$ point near $E_{F}$ taken at two representative temperatures (the color bar is shown at the top). (b,c) Corresponding integrated EDC (b) and MDC at $E_{F}$ (c).

spectra near $E_{\mathrm{F}}$. It is clear that the $J=5 / 2 \mathrm{KR}$ is extremely weak (if any), and therefore there must be a different cause for the FS expansion. In fact, Fig. 2(a) shows that the electron pocket expansion is accompanied by a downward shift of the whole Ce $5 d$ band in energy. This behavior can be most naturally explained by a slight increase of Ce valence on cooling from $\sim 35$ to $\sim 10 \mathrm{~K}$, which we estimate to be $\sim 0.01$ based on the Luttinger count of the observed electron pocket. As stated in the Introduction, such temperature dependence of the RE valence in KL systems appears to be quite natural $[6,37,38]$. In low carrier density systems, the many-body Kondo effect is largely diminished due to the stringent requirement for the conduction electron density and, therefore, the valence fluctuation originating in the substantial $c$ - $f$ mixing can be resolved via the FS expansion. Such a small valence-induced change in FS is difficult to resolve in metallic KL systems, but it can be identified in low carrier density KL systems.

The valence-induced pocket expansion is mainly along the short axis of the ellipse [Fig. 1(e)], demonstrating that the $c-f$ mixing is highly anisotropic in momentum space. While we do not have a good quantitative explanation yet, this is likely related to the hybridization matrix element involving the detailed wave function of $\mathrm{Ce} 4 f$ and conduction electrons. In addition to the downward shift of the Ce $5 d$ band near $E_{\mathrm{F}}$ at the $\bar{M}$ point [Fig. 2(a)], we also observe a similar shift of the bands at deeper binding energies at low temperature (see the Supplemental Material [28] for detailed EDC analysis). These deeper bands contain contributions from Ce $5 d$ orbitals as well, although mixing with $\mathrm{Bi} 6 p$ orbitals is also present due to band overlapping [27]. These bands can therefore shift toward lower energies as a result of the Ce valence change.

The observed intensity decrease of the $4 f^{0}$ peak in Fig. 3(c) is much larger than the estimated valence increase of $\sim 0.01$ based on the Luttinger count of the electron pocket. This indicates that the observed spectral weight transfer in Fig. 3(c) should not be interpreted as being directly proportional to

the actual number of transferred electrons. Instead, since the resonant ARPES intensity is proportional to the product of the electronic spectral function and the photoemission matrix element from the $f$ component (due to the resonance condition), the spectral weight transfer in Fig. 3(c) should contain a large contribution from the variation of the matrix element, i.e., the reduced (increased) $f$ component in the wave function of the $4 f^{0}$ state (conduction bands) upon cooling.

The proposed $p$ - $f$ mixing between the $\mathrm{Bi} 6 p$ and $\mathrm{Ce} 4 f$ states apparently does not change much within the measurement temperature window in $\mathrm{CeBi}$ [17], as evidenced by the weak $J=5 / 2 \mathrm{KR}$ and the lack of any resolvable change in the Bi $6 p$ band [see Figs. 4(b) and 4(c)]. On the other hand, the large temperature-dependent expansion of the $\mathrm{Ce}$ $5 d$ band can be explained by a tiny increase of the Ce valence $(\sim 0.01)$, which could be checked by ultrahigh-resolution XAS at the Ce $L$ edge in the future $[39,40]$. The expansion of the Ce $5 d$ pocket provides evidence for a strong covalent $d-f$ mixing among neighboring $\mathrm{Ce}$ ions, which contributes to the observed spectral transfer from the localized Ce $4 f$ states into itinerant $\mathrm{Ce} 5 d$ bands near $E_{F}$, i.e., the action of valence fluctuations. It is interesting to compare our results on $\mathrm{CeBi}$ with those of $\mathrm{CeSb}$, where a recent ARPES study also revealed two different channels of interactions by the $\mathrm{Ce}$ $4 f$ electrons [29], i.e., the Kondo interaction (related to $p$ - $f$ mixing) and magnetic exchange interaction (via $d$ - $f$ mixing). We make the interesting observation that the $d$ - $f$ mixing is obviously stronger in $\mathrm{CeBi}$, compared to $\mathrm{CeSb}$, with no sign of magnetic exchange splitting [29]. This difference is likely related to differences in the lattice constants and/or charge carrier densities.

\section{CONCLUSIONS}

To summarize, we have experimentally observed a large expansion (by $\sim 40 \%$ ) of the Ce $5 d$ pocket in $\mathrm{CeBi}$ at low temperature, while the $\mathrm{Bi} 6 p$ pockets remain unchanged. Resonant ARPES measurements at low temperature revealed pronounced spectral weight transfer from the $4 f^{0}$ state into the conduction bands near $E_{F}$, sharing similar temperature dependence with the FS expansion. The $J=7 / 2$ satellite of the KR could be clearly resolved, implying that the Kondo screening is operating, although a weak $J=5 / 2 \mathrm{KR}$ at $E_{\mathrm{F}}$ indicates very low $T_{K}$. The observed FS expansion, distinct from the small-to-large FS change in metallic KL systems, can be explained by a small valence change of $\mathrm{Ce}(\sim 0.01)$. Such valence-induced FS expansion goes beyond a simple rigid band shift. Rather, it is highly anisotropic in momentum space, due to an anisotropic $c$ - $f$ mixing; there is strong momentum-dependent hybridization of the Ce $4 f$ electrons with the $5 d$ electrons of neighboring $\mathrm{Ce}$ ions, but an only very weak one with the Bi $6 p$ electrons. Our study demonstrates that weak valence fluctuations can be made visible in low carrier density KL systems via significant thermally driven changes in the FS, which can be important in understanding relevant physical properties. The observed concurrence of real and virtual charge fluctuations as well as local-moment AFM order describes a state of quantum matter which has been enigmatic for years and deserves further scrutiny by future studies. 


\section{ACKNOWLEDGMENTS}

This work is supported by National Key R\&D Program of the MOST of China (Grants No. 2017YFA0303100 and No. 2016YFA0300203), National Science Foundation of China (Grants No. 11674280 and No. 11274006), and the Fundamental Research Funds for the Central Universities (Grant No. 2019QNA3007). We would like to thank Pengdong Wang, Dr. Caizhi $\mathrm{Xu}$, and Dr. Junzhang Ma for help in the synchrotron ARPES measurements. Y.L. thanks Prof. Qimiao Si and Prof.
Stefan Kirchner for enlightening discussions. The synchrotron ARPES measurements were performed at BL13U beamline in Hefei National Synchrotron Radiation Laboratory, SIS beamline at Swiss Light Source, and 29-ID IEX beamline at Advanced Photon Source (APS). APS is supported by the US Department of Energy (DOE) Office of Science under Contract No. DE-AC02-06CH11357; additional support by the National Science Foundation under Grant No. DMR-0703406 is also acknowledged.
[1] P. Coleman, Heavy fermions: electrons at the edge of magnetism, in Handbook of Magnetism and Advanced Magnetic Materials (Wiley, New York, 2007), Vol. 1.

[2] H. v. Löhneysen, A. Rosch, M. Vojta, and P. Wölfle, Rev. Mod. Phys. 79, 1015 (2007).

[3] Q. Si and F. Steglich, Science 329, 1161 (2010).

[4] F. Steglich, J. Aarts, C. D. Bredl, W. Lieke, D. Meschede, W. Franz, and H. Schäfer, Phys. Rev. Lett. 43, 1892 (1979).

[5] H. Q. Yuan, F. M. Grosche, M. Deppe, C. Geibel, G. Sparn, and F. Steglich, Science 302, 2104 (2003).

[6] A. C. Hewson, The Kondo Problem to Heavy Fermions (Cambridge University Press, Cambridge, 1997).

[7] D. Wohlleben and B. Wittershagen, Adv. Phys. 34, 403 (1985).

[8] W. Franz, F. Steglich, W. Zell, D. Wohlleben, and F. Pobell, Phys. Rev. Lett. 45, 64 (1980).

[9] Y. Hiranaka, A. Nakamura, M. Hedo, T. Takeuchi, A. Mori, Y. Hirose, K. Mitamura, K. Sugiyama, M. Hagiwara, T. Nakama, and Y. Ōnuki, J. Phys. Soc. Jpn. 82, 083708 (2013).

[10] Y. Luo, F. Ronning, N. Wakeham, X. Lu, T. Park, Z.-A. Xu, and J. D. Thompson, Proc. Natl. Acad. Sci. USA 112, 13520 (2015).

[11] S. Dzsaber, L. Prochaska, A. Sidorenko, G. Eguchi, R. Svagera, M. Waas, A. Prokofiev, Q. Si, and S. Paschen, Phys. Rev. Lett. 118, 246601 (2017).

[12] C. Y. Guo, F. Wu, Z. Z. Wu, M. Smidman, C. Cao, A. Bostwick, C. Jozwiak, E. Rotenberg, Y. Liu, F. Steglich, and H. Q. Yuan, Nat. Commun. 9, 4622 (2018).

[13] P.-Y. Chang, O. Erten, and P. Coleman, Nat. Phys. 13, 794 (2017).

[14] K. Kuroda, M. Ochi, H. S. Suzuki, M. Hirayama, M. Nakayama, R. Noguchi, C. Bareille, S. Akebi, S. Kunisada, T. Muro, M. D. Watson, H. Kitazawa, Y. Haga, T. K. Kim, M. Hoesch, S. Shin, R. Arita, and T. Kondo, Phys. Rev. Lett. 120, 086402 (2018).

[15] T. Suzuki, Phys. B (Amsterdam, Neth.) 186-188, 347 (1993).

[16] H. Bartholin, P. Burlet, S. Quezel, J. Rossat-Mignod, and O. Vogt, J. Phys. Colloq. 40, C5-130 (1979).

[17] H. Takahashi and T. Kasuya, J. Phys. C: Solid State Phys. 18, 2697 (1985).

[18] G.-J. Hu and B. R. Cooper, Phys. Rev. B 38, 9127 (1988).
[19] H. Kumigashira, S.-H. Yang, T. Yokoya, A. Chainani, T. Takahashi, A. Uesawa, T. Suzuki, O. Sakai, and Y. Kaneta, Phys. Rev. B 54, 9341 (1996).

[20] M. S. Litsarev, I. Di Marco, P. Thunström, and O. Eriksson, Phys. Rev. B 86, 115116 (2012).

[21] O. Sakai and Y. Shimizu, J. Phys. Soc. Jpn. 76, 044707 (2007).

[22] T. Kasuya, Y. S. Kwon, T. Suzuki, K. Nakanishi, F. Ishiyama, and K. Takegahara, J. Magn. Magn. Mater. 90-91, 389 (1990).

[23] T. Kasuya, Y. Hasa, Y. S. Kwon, and T. Suzuki, Phys. B (Amsterdam, Neth.) 186, 9 (1993).

[24] G. Kresse and J. Hafner, Phys. Rev. B 47, 558 (1993).

[25] X. Duan, F. Wu, J. Chen, P. Zhang, Y. Liu, H. Q. Yuan, and C. Cao, Commun. Phys. 1, 71 (2018).

[26] F. Tran and P. Blaha, Phys. Rev. Lett. 102, 226401 (2009).

[27] P. Li, Z. Wu, F. Wu, C. Cao, C. Guo, Y. Wu, Y. Liu, Z. Sun, C.-M. Cheng, D.-S. Lin, F. Steglich, H. Yuan, T.-C. Chiang, and Y. Liu, Phys. Rev. B 98, 085103 (2018).

[28] See Supplemental Material at http://link.aps.org/supplemental/ 10.1103/PhysRevB.100.155110 for additional ARPES data and analysis.

[29] S. Jang, R. Kealhofer, C. John, S. Doyle, J. Hong, Q. Si, O. Erten, J. D. Denlinger, and J. G. Analytis, Sci. Adv. 5, eaat7158 (2019).

[30] D. Ehm, S. Hüfner, F. Reinert, J. Kroha, P. Wölfle, O. Stockert, C. Geibel, and H. V. Löhneysen, Phys. Rev. B 76, 045117 (2007).

[31] J. W. Allen, J. Phys. Soc. Jpn. 74, 34 (2005).

[32] J. H. Shim, K. Haule, and G. Kotliar, Science 318, 1615 (2007).

[33] S.-K. Mo, W. S. Lee, F. Schmitt, Y. L. Chen, D. H. Lu, C. Capan, D. J. Kim, Z. Fisk, C.-Q. Zhang, Z. Hussain, and Z.-X. Shen, Phys. Rev. B 85, 241103(R) (2012).

[34] S.-I. Fujimori, Y. Saitoh, T. Okane, A. Fujimori, H. Yamagami, Y. Haga, E. Yamamoto, and Y. Ōnuki, Nat. Phys. 3, 618 (2007).

[35] Q. Y. Chen, D. F. Xu, X. H. Niu, J. Jiang, R. Peng, H. C. Xu, C. H. P. Wen, Z. F. Ding, K. Huang, L. Shu, Y. J. Zhang, H. Lee, V. N. Strocov, M. Shi, F. Bisti, T. Schmitt, Y. B. Huang, P. Dudin, X. C. Lai, S. Kirchner, H. Q. Yuan, and D. L. Feng, Phys. Rev. B 96, 045107 (2017).

[36] S. Jang, J. D. Denlinger, J. W. Allen, V. S. Zapf, M. B. Maple, J. N. Kim, B. G. Jang, and J. H. Shim, arXiv:1704.08247v1. 
[37] L. Moreschini, C. Dallera, J. J. Joyce, J. L. Sarrao, E. D. Bauer, V. Fritsch, S. Bobev, E. Carpene, S. Huotari, G. Vankó, G. Monaco, P. Lacovig, G. Panaccione, A. Fondacaro, G. Paolicelli, P. Torelli, and M. Grioni, Phys. Rev. B 75, 035113 (2007).

[38] K. Kummer, C. Geibel, C. Krellner, G. Zwicknagl, C. Laubschat, N. B. Brookes, and D. V. Vyalikh, Nat. Commun. 9, 2011 (2018).
[39] M. Sundermann, F. Strigari, T. Willers, H. Winkler, A. Prokofiev, J. M. Ablett, J.-P. Rueff, D. Schmitz, E. Weschke, M. M. Sala, A. A. Zein, A. Tanaka, M. W. Haverkort, D. Kasinathan, L. H. Tjeng, S. Paschen, and A. Severing, Sci. Rep. 5, 17937 (2015).

[40] J.-P. Rueff, S. Raymond, M. Taguchi, M. Sikora, J.-P. Itié, F. Baudelet, D. Braithwaite, G. Knebel, and D. Jaccard, Phys. Rev. Lett. 106, 186405 (2011). 